Fabrizio Anella(i)

\title{
Rational curves on genus-one fibrations
}

Received: 18 July 2019 / Accepted: 30 October 2020 /

Published online: 17 November 2020

Abstract. In this paper we look for necessary and sufficient conditions for a genus-one fibration to have rational curves. We show that a projective variety with log terminal singularities that admits a relatively minimal genus-one fibration $X \rightarrow B$ does contain vertical rational curves if and only if it not isomorphic to a finite étale quotient of a product $\tilde{B} \times E$ over $B$. Many sufficient conditions for the existence of rational curves in a variety that admits a genus-one fibration are proved in this paper.

\section{Introduction}

The starting point of this work has been the following folklore conjecture.

Conjecture 0.1. Every (possibly singular) Calabi-Yau variety does contain rational curves.

This conjecture is unsolved even for smooth Calabi-Yau manifolds in dimension three. We started studying Calabi-Yau varieties that admit a genus-one fibration and we got a positive answer in [2, Theorem 1]. At this point it is natural to ask the following question.

Question 0.2. Under which conditions a genus-one fibration does contain rational curves?

Without asking anything on the base of the fibration, we can say something only on the rational curves that are vertical for the fibration. The main purpose of this article is to prove the following answer to Question 0.2 that gives a complete characterization in the case of relatively minimal genus-one fibration.

Theorem 0.3. Let $X \stackrel{\pi}{\rightarrow} B$ be genus-one fibration such that $K_{X} \sim_{\mathbb{Q}} \pi^{*} L$ for some $\mathbb{Q}$-Cartier $\mathbb{Q}$-divisor on B. Then $X$ does not contain vertical rational curves if and only if there exists a finite cover $\tilde{B}$ of $B$ and a genus-one curve $E$ such that $X$ is a finite étale quotient of $\tilde{B} \times E$ over $B$.

F. Anella (凶): Dipartimento di Matematica e Fisica, Università Roma 3, Largo San Leonardo Murialdo 1, 00146 Rome, Italy. e-mail: fabrizio.anella2@uniroma3.it; anella@math.unibonn.de

Present Address: F. Anella: Mathematisches institut, Universität Bonn, Endenicher Allee 60, 53115 Bonn, Germany

Mathematics Subject Classification: Primary: 14J32 · Secondary: 14E30, 14D06 
A key ingredient for the proof of this result is the proof of the following theorem, that is a generalization of [2, Theorem 1] and of [7, Theorem 1.1].

Theorem 0.4. Let $(X, \Delta)$ be a klt pair such that there exists a surjective morphism $\phi: X \rightarrow B$ to a variety of dimension $n-1$. Suppose moreover $K_{X}+\Delta \sim_{\mathbb{Q}} \phi^{*} L$ for some $\mathbb{Q}$-Cartier $\mathbb{Q}$-divisor $L$ on $B$ and the augmented irregularity of $X$ is zero, then there exists a subvariety of $X$ of dimension $n-1$ covered by rational curves contracted by $\phi$.

This theorem has some interesting consequences, like the following.

Corollary 0.5. Let $X$ be a projective variety of dimension $n$ with log terminal singularities, $\kappa(X)=n-1$ and $\tilde{q}(X)=0$. The variety $X$ does contain rational curves.

Using a very nice result of Lazić and Peternell [20, Theorem 6.12] we can do slightly better in the smooth case.

Corollary 0.6. Let $X$ be a smooth projective variety with $\tilde{q}(X)=0$. If $X$ is covered by genus-one curves, then it contains a rational curve.

In Sect. 1 we fix the notation and recall some definitions used in the subsequent parts. In Sect. 2 we discuss some properties of the augmented irregularity. In Sect. 3 we prove Theorem 0.4 . The proof is organized in several lemmas which are of independent interest. In Sect. 4 we explain some generalizations and consequences of Theorem 0.4. There will be a more accurate analysis of Question 0.2 in the forthcoming Ph.D. thesis of the author.

\section{Preliminaries}

In this paper every variety will be an irreducible normal quasi-projective variety over the complex numbers. The variety $X$ will be always projective and of dimension $n \geq 2$. The notation and standard properties about singularities that are used in this article can be found for example in [18]. For the reader's convenience, we recall some definitions.

Definition 1.1. Let $\phi: X \rightarrow B$ be a surjective morphism of normal projective varieties and $D \in \mathrm{WDiv}(X)$ be a prime Weil divisor. We say that $D$ is exceptional if $\operatorname{cod}_{B}(\phi(D)) \geq 2$. We say that $D$ is of insufficient fiber type if $\operatorname{cod}_{B}(\phi(D))=1$ and there exists another prime Weil divisor $D^{\prime} \neq D$ such that $\phi\left(D^{\prime}\right)=\phi(D)$. In either of the above cases, we say that $D$ is degenerate.

Definition 1.2. A morphism $f: Z \rightarrow Y$ between normal varieties is called quasiétale if $f$ is quasi-finite and étale in codimension one. The augmented irregularity of a projective variety $Y$ is the following supremum

$$
\tilde{q}(Y):=\sup \{q(Z) \mid Z \rightarrow Y \text { is a finite quasi-étale cover }\} .
$$

Remark 1.3. The above definition of quasi-étale morphism is not the same of [4]. 
Definition 1.4. Let $f: X \rightarrow Y$ be a surjective morphism between normal varieties. The set of singular values of $f$ is the following subset of $Y$

$$
\operatorname{Sv}(f)=\left\{y \in Y \mid \operatorname{dim}\left(f^{-1}(y)\right)>\operatorname{dim}(X)-\operatorname{dim}(Y) \text { and } f^{-1}(y) \text { is singular }\right\} .
$$

One can associate to any elliptic curve a complex number called its $j$-invariant. This association is modular, which means that a genus-one fibration $f: Y \rightarrow B$ comes with a rational map $j: B \rightarrow \mathbb{C}$ called $j$-function that is at least defined over the smooth values of $f$. Good references for standard facts about the $j$-map of an elliptic fibration are [12, Chap. 4, Sect. 26] and [16].

Definition 1.5. A projective variety with klt singularities $Y$ (resp. a klt pair $(Y, \Delta)$ ) is a Calabi-Yau variety (resp. a log Calabi-Yau) if $K_{Y} \sim_{\mathbb{Q}} 0$ (resp. $K_{Y}+\Delta \sim_{\mathbb{Q}} 0$ ) and $\tilde{q}(Y)=0$.

In some recent works on Beauville-Bogomolov decomposition (see for example $[8,9,14])$ several definitions of Calabi-Yau varieties appeared. In our definition we include also products of Calabi-Yau and irreducible holomorphic symplectic varieties in the sense of [9, Definition 8.16].

Definition 1.6. A projective variety with klt singularities $Y$ (resp. a pair $(Y, \Delta)$ ) together with a fibration $Y \stackrel{f}{\rightarrow} B$ is a Calabi-Yau fiber space (resp. a log CalabiYau fiber space) if the general fiber $Y_{t}$ of $f$ (resp. the pairs $\left.\left(Y_{t}, \Delta_{t}\right)\right)$ has $\mathbb{Q}$-linearly trivial canonical bundle (resp. $K_{Y_{t}}+\Delta_{t} \sim_{\mathbb{Q}} 0$ ).

When the general fiber is a curve, we are mainly interested in the case where the boundary $\Delta$ does not intersect the general fiber. Indeed if the intersection is non-trivial, then $Y$ is uniruled.

Definition 1.7. A genus-one fibration is a morphism $\pi: Y \rightarrow B$ between normal projective varieties with connected fibers and such that the general fiber is a smooth genus-one curve.

A particular case of Calabi-Yau fiber space is the following.

Definition 1.8. A Calabi-Yau fiber space $Y \stackrel{f}{\rightarrow} B$ (resp. a log Calabi-Yau fiber space $(Y, \Delta) \stackrel{f}{\rightarrow} B$ ) is called a relatively minimal (resp. log) Calabi-Yau fiber space if $K_{Y} \sim_{\mathbb{Q}} f^{*} L\left(K_{Y}+\Delta \sim_{\mathbb{Q}} f^{*} L\right)$ for some $\mathbb{Q}$-Cartier $\mathbb{Q}$-divisor on $B$.

In particular when we say that a genus-one fibration is relatively minimal, we are saying that $X$ has $\log$ terminal singularities.

An important class of examples of Calabi-Yau fiber spaces is given by orbibundles. We just recall the construction of orbibundles because they play a key role in the proof of Theorem 0.4. More properties and details can be found in the article of Kollár [17, Sect. 7].

Let $\tilde{B}$ be a normal variety, $F$ a variety with klt singularities, $K_{F} \sim_{\mathbb{Q}} 0$ and $\tilde{Y}:=\tilde{B} \times F$ their product. Let $G$ be a finite group and $\rho_{B}: G \rightarrow \operatorname{Aut}(\tilde{B})$, $\rho_{F}: G \rightarrow \operatorname{Aut}(F)$, two faithful representations.

Definition 1.9. An orbibundle is the Calabi-Yau fiber space

$$
(Y \rightarrow B):=\tilde{Y} / G \rightarrow \tilde{B} / G
$$

obtained as quotient with respect to the diagonal representation of $G$. 


\section{Some remarks on the augmented irregularity}

It is not difficult to show that the augmented irregularity is a birational invariant for smooth projective varieties. To show this fact, take two smooth birational projective varieties $X$ and $X^{\prime}$. We can suppose, considering a resolution of the birational map, that there is a well-defined morphism $X^{\prime} \rightarrow X$.

Any quasi-étale cover $Z$ of $X$ (hence globally étale) can be pulled back to an étale cover $Z^{\prime}$ of $X^{\prime}$. These two covers are smooth and birational, so $q(Z)=q\left(Z^{\prime}\right)$. Since this argument works for any quasi-étale cover of $X$, taking the sup we get $\tilde{q}(X) \leq \tilde{q}\left(X^{\prime}\right)$.

To show the other inequality, let $Z^{\prime}$ be a quasi-étale cover of $X^{\prime}$. This variety has a natural map to $X$. Passing through the Stein factorization of this map, one can conclude as for the other inequality.

However the augmented irregularity is not a birational invariant for projective varieties with canonical singularities. Indeed the standard construction of a Kummer surface is a counterexample.

Example 2.1. We take an elliptic curve $E$ and then consider the quotient $X:=$ $(E \times E) / \pm$ of the product of two copies of $E$ by the involution. The quotient map $E \times E \rightarrow X$ is a quasi-étale cover, so $\tilde{q}(X) \geq 2$. Moreover by [8, Remark 4.3] it holds the equality. However a minimal resolution $\tilde{X}$ of $X$ is a K3 surface. In particular $\tilde{X}$ is simply connected, hence $\tilde{q}(\tilde{X})=0$. The variety $X$ is thus also an example of a regular variety with non-trivial augmented irregularity.

However, it follows from the last part of the proof of Theorem 0.4 that the augmented irregularity is invariant for birational morphisms that are isomorphism in codimension one of projective varieties with klt singularities.

It is natural to ask whether there exists some manageable conditions for the vanishing of the augmented irregularity of a variety. It is easy to check [2, Remark $3]$ that a variety $X$, say smooth for simplicity, with finite fundamental group has $\tilde{q}(X)=0$. For varieties with numerically trivial canonical divisor, an interesting characterization is given in [9, Theorem 11.1], where the authors proved that, in this setting, $\tilde{q}(X)=0$ if and only if for any $k>0$ there are no non-trivial symmetric reflexive forms, i.e. $H^{0}\left(X, \operatorname{Sym}^{[k]} \Omega_{X}^{1}\right)=0 \forall k>0$. We prove that an implication still holds without the assumption on the canonical bundle: the following is a sufficient condition for the vanishing of the augmented irregularity which does not rely on computations of invariants on quasi-étale covers, but only on invariants of the variety under investigation.

Proposition 2.2. Let $X$ be a projective variety with log terminal singularities. If $H^{0}\left(X, \operatorname{Sym}^{[k]} \Omega_{X}^{1}\right)=0$ for every $k>0$, then $\tilde{q}(X)=0$.

Let us recall that by $\operatorname{Sym}^{[k]} \Omega_{X}^{1}$ we mean the sheaf of the reflexive symmetric $k$-forms on $X$.

Proof. Suppose, by contradiction, that there is a quasi-étale cover $\tilde{X} \rightarrow X$ with $H^{1}\left(\tilde{X}, \mathcal{O}_{\tilde{X}}\right) \neq 0$. The variety $\tilde{X}$ is klt $[18$, Proposition 5.20$]$ and by duality [10, Proposition 6.9] there is a non-zero reflexive form $\omega \in H^{0}\left(\tilde{X}, \Omega_{\tilde{X}}^{[1]}\right)$. By definition, 
the sections of a reflexive sheaf on $X$ are exactly the sections on the regular part of $X$. So to construct a non-zero global section of $\operatorname{Sym}^{[k]} \Omega_{X}^{1}$, we construct an element in $H^{0}\left(X_{\text {reg }}\right.$, Sym $\left.^{k} \Omega_{X_{\text {reg }}}^{1}\right)$. Now we consider just the restriction to the regular locus of $X$ :

$$
Y:=\tilde{X} \times_{X} X_{\text {reg }} \rightarrow X_{\text {reg }}
$$

This is a finite étale cover, so we can find a further étale cover, finite over the regular part $\tilde{Y} \rightarrow X_{\text {reg }}$, that is Galois. Let $G$ be the group of deck transformations of $\tilde{Y}$ over $X_{\text {reg. }}$. By abuse of notations we call again $\omega$ the pullback to $\tilde{Y}$ of $\omega$. Now consider the section $\tilde{\alpha}:=\sum_{\tau \in G} \otimes_{\rho \in G} \tau^{*} \rho^{*} \omega \in H^{0}\left(\tilde{Y},\left(\Omega_{\tilde{Y}}^{1}\right)^{\otimes N}\right)$, where $N=|G|$. This section is invariant under the action of the deck trasformations, so it descends to a section $\alpha$ of $H^{0}\left(X_{\text {reg }},\left(\Omega_{X_{\text {reg }}}^{1}\right)^{\otimes N}\right)$. By construction it is easy to check that this section is symmetric, i.e. $\omega$ belongs to $H^{0}\left(X_{\text {reg }}, \operatorname{Sym}^{N}\left(\Omega_{X_{\text {reg }}}^{1}\right)\right)$. It is less trivial to prove that $\tilde{\alpha}$, and hence $\alpha$, is non-zero.

For any non-zero element $\gamma \in H^{0}\left(\tilde{Y}, \Omega_{\tilde{Y}}^{1}\right)$ and a general point $p \in \tilde{Y}$ the space $\operatorname{Ker}(\gamma) \subset T_{\tilde{Y}, p}$ is a proper subspace. Since $\omega \neq 0$ and the elements $\rho \in G$ are automorphisms (and we are working over $\mathbb{C}$ ), also $\rho^{*} \omega$ are non-zero elements in $H^{0}\left(\tilde{Y}, \Omega_{\tilde{Y}}^{1}\right)$. So for general $p \in \tilde{Y}$ we can choose a tangent vector

$$
0 \neq v \in T_{\tilde{Y}, p} \backslash \bigcup_{\rho \in G} \operatorname{Ker}\left(\left(\rho^{*} \omega\right)_{p}\right)
$$

Now we can evaluate our section $\tilde{\alpha}$ at the vector $v^{\otimes N}$. The computations are the following:

$$
\tilde{\alpha}\left(v^{\otimes N}\right)=\sum_{\tau \in G} \bigotimes_{\rho \in G} \tau^{*} \rho^{*} \omega(v)=\sum_{\tau \in G} \prod_{\rho \in G} \tau^{*} \rho^{*} \omega(v)=N \prod_{\rho \in G} \rho^{*} \omega(v) \neq 0 .
$$

So we have constructed a non-zero section of $\left.H^{0}\left(X_{\text {reg }}, \operatorname{Sym}^{N} \Omega_{X_{\text {reg }}}^{1}\right)\right)$ that corresponds to a non-zero section of $H^{0}\left(X, \operatorname{Sym}^{[N]} \Omega_{X}^{1}\right)$.

\section{Proof of the main theorem}

We start with a lemma that is certainly well-known to the experts.

Lemma 3.1. Let $X \stackrel{\pi}{\rightarrow}$ B be a genus-one fibration. If the subvariety of the singular values $\operatorname{Sv}(\pi) \subset B$ has codimension at least two in $B$, then the family $\pi$ is isotrivial.

For the proof of this result we refer to [2, Lemma 3]

Now we study the general fibers over the singular values of a genus-one fibration.

Lemma 3.2. Let $\phi: X \rightarrow B$ be a genus-one fibration and $Z:=\operatorname{Sv}(\phi)$. Suppose that $\operatorname{cod}_{B}(Z)=1$, then a general fiber over $Z$ is $m E+\sum m_{i} R_{i}$ where $E$ is a genus-one curve and $R_{i}$ are rational curves. 
Proof. We can study the restriction of $\phi$ to a surface as follows. Let $H$ be a very ample divisor on $B$ such that $(n-2) H+L$ is globally generated. The pullback $\phi^{*} H$ is a globally generated Cartier divisor. Moreover there is an isomorphism

$$
H^{0}\left(X, \phi^{*} H\right) \simeq H^{0}\left(B, \phi_{*}\left(\phi^{*} H\right)\right) \simeq H^{0}(B, H)
$$

because $\phi$ has connected fibers. This implies that general elements in $|H|$ are general also in $\left|\phi^{*}(H)\right|$. So we choose $n-2$ general divisors $D_{1}, \ldots, D_{n-2} \in|H|$ such that $C:=D_{1} \cap \ldots \cap D_{n-2}$ is a smooth irreducible curve in $B_{\text {reg not contained }}$ in the locus of singular values of $\phi$ and $S:=\phi^{-1}\left(D_{1}\right) \cap \ldots \cap \phi^{-1}\left(D_{n-2}\right)$ is a normal surface. Passing to a resolution and looking at the Kodaira's table [3, Sect. V.7], it is easy to check that the singular fibers of $\left.\phi\right|_{S}$ are $m E+\sum m_{i} R_{i}$ where $E$ is an elliptic curve and $R_{i}$ are rational curves. The condition on the dimension of $Z$ ensures that a general point in $Z$ lies on a curve obtained as a general complete intersection of hyperplane sections.

Remark 3.3. If $X$ contains no uniruled codimension one subvarieties but $\operatorname{Sv}(\phi)$ has codimension one in $B$, then the fibers over any generic point of $\operatorname{Sv}(\phi)$ of dimension $n-2$ is a multiple genus-one curve.

Remark 3.4. It follows from Lemma 3.2 and from a result of Kawamata [15], that a relatively minimal genus-one fibration with no codimension one subvarieties covered by vertical rational curves, has no degenerate divisors.

Lemma 3.2 can be seen as a soft version of Kodaira's table in higher dimension. With the same strategy of the proof of this lemma one can certainly do a better classification of singular fibers. Using the techniques of Lemma 3.2 we can control only the general singular fiber in codimension one. Other fibers may appear in greater codimension. Now we can merge together these lemmas and prove the following result.

Lemma 3.5. Let $X \stackrel{\phi}{\rightarrow} B$ be a genus-one fibration between normal varieties such that $X$ does not contain codimension one subvarieties that are covered by vertical rational curves. Then the family $X \stackrel{\phi}{\rightarrow} B$ is isotrivial.

This lemma should be compared with [20, Proposition 6.5] and [21, Proof of Corollary 3.34].

Proof of Lemma 3.5. We can assume by Lemma 3.1, that $Z:=\operatorname{Sv}(\phi))$ has codimension one in $B$. The general fibers over $Z$ are classified in Lemma 3.2. In the general fibers over $Z$ there are only multiple genus-one curves.

Now we can proceed cutting with hyperplane sections as in the proof of Lemma 3.2. In this way we get many curves $C$ in $B$, with only genus-one fibers (possibly multiple) over them. Up to consider a finite possibly ramified base change, we can assume this map has a section. The $j$-invariant for multiple elliptic curves is welldefined as one can easily check with a semistable reduction. Since the curve $C$ is complete this implies that the $j$-map is constant, i.e. the family $\pi$ restricted over $C$ is isotrivial. 
For each curve $C$ obtained in this way we get an isotrivial family. From this fact it follows that the all the family over $B$ is isotrivial. Let us prove this fact by induction on the dimension of $B$.

There is nothing to prove if the dimension is one. By induction, we can suppose that the family is isotrivial when restricted to an ample subvariety $H \subset B$. The fibers over curves that are general complete intersections are pairwise isomorphic and these curves must intersect $H$. The union of these curves dominates $B$. This implies that the family $X \stackrel{\phi}{\rightarrow} B$ is isotrivial.

Another way to prove this lemma is to consider the $j$-map directly from $B$.

Before going through the proof of Theorem 0.4 , we state another lemma that will be useful in the proof.

Lemma 3.6. Let $Y$ and $Y^{\prime}$ be projective varieties of dimension n, with two fibrations $f$ and $f^{\prime}$ to a normal variety $B$ of dimension $n-1$. Suppose both fibrations have no degenerate divisors and they are birational via a rational map $g$. Then $g$ contracts some divisors if and only if $g^{-1}$ does.

Proof. Suppose $D \subset Y$ is a prime divisor contracted in $Y^{\prime}$ by $g$ and that $f$ has no degenerate divisors. Let us observe that a rational map between normal varieties is always defined in codimension one, so we can decide if a divisor is contracted also for rational map. If $f(D)=B$ then, by dimensional reasons, $\operatorname{dim}(g(D))=n-1$. The divisor $D$ is not $f$-exceptional, so $f(D)$ is a divisor in $B$. By dimensional reasons, its preimage $f^{\prime-1}$ in $X^{\prime}$ contains a Weil divisor $W$. This divisor is not the image of a Weil divisor of $Y$ because we are supposing the only divisor in $f^{-1}(f(D))$ is $D$ that is contracted by $g$. With this in mind, it follows easily that $g^{-1}$ contracts $W$.

Finally we can proceed with the proof of Theorem 0.4 . For the reader's convenience, we state again the result that we are going to prove.

Theorem 3.7. Let $(X, \Delta) \stackrel{\phi}{\rightarrow} B$ be a relatively minimal log Calabi-Yau fiber space such that $\tilde{q}(X)=0$ and $\operatorname{dim}(B)=n-1$. Then there exists a subvariety of $X$ of dimension $n-1$ covered by rational curves contracted by $\phi$.

Proof. Since $K_{X}+\Delta \sim \sim_{\mathbb{Q}} \phi^{*} L$, the restriction of $K_{X}+\Delta$ to a general fiber of $\phi$ is $\mathbb{Q}$-linearly trivial. A general fiber of $\phi$ is a smooth curve contained in the smooth locus of $X$, so by adjunction formula $\left.K_{X_{t}} \sim K_{X}\right|_{X_{t}}$. This implies that $K_{X_{t}} \sim \mathbb{Q}-\left.\Delta\right|_{X_{t}}$ and hence the general fiber has genus at most one. If the genus is zero the variety $X$ is uniruled, so we can suppose $\phi$ is a genus-one fibration. Note that even if $\Delta$ and $K_{X}$ are not $\mathbb{Q}$-Cartier $\mathbb{Q}$-divisors their restriction on a neighborhood of a general fiber $X_{t}$ is $\mathbb{Q}$-Cartier.

If the fibration is non-isotrivial then, by Lemma 3.5, there exists a divisor in $X$ covered by vertical rational curves. It remains to study the case $\phi$ is an isotrivial genus-one fibration without exceptional divisors. Under these conditions by a result of Kollár [17, Theorem 44 (1)] $X$ is birational over $B$ to an orbibundle $X_{\text {orb }}$. Let us recall that $X_{\text {orb }}$ is a finite quotient of $\tilde{X}:=\tilde{B} \times F$, where $F$ is a general fiber of $\phi$ and $\tilde{B}$ is a finite cover of $B$. 
We claim that we can assume $X$ and $X_{\text {orb }}$ are isomorphic in codimension one. Indeed by construction the variety $X_{\text {orb }}$ has no $\phi$-degenerate divisors. Since $\phi$ is a relatively minimal Calabi-Yau fiber space, by [1, Theorem 0.2] there exists some boundary $\Delta$ on $B$ such that $(B, \Delta)$ is klt. By [18, Proposition 5.20] also $\left(X_{\text {orb }}, \Delta^{\prime}\right)$ is klt for some boundary. As easy consequence of [13, Corollary 1.6], a divisor in $X$ contracted by the birational map in $X_{\text {orb }}$ is covered by rational curves. Finally the claim follows from Lemma 3.6.

Since $\tilde{X} \rightarrow X_{\text {orb }}$ is quasi-étale [17, see Lemma 38], there exists an open subset $\tilde{X}^{\circ} \subset \tilde{X}$ such that the induced morphism $\tilde{X}^{\circ} \rightarrow X$ is quasi-finite, étale and $\operatorname{cod}_{\tilde{X}}\left(\tilde{X}^{\circ}\right) \geq 2$. By Zariski's Main Theorem [11, Théorème (4.4.3)] a quasi-finite morphism factorizes through an open immersion and a finite morphism, so there exists a commutative diagram

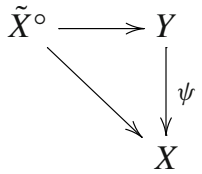

with $Y$ projective and the morphism $\psi$ is étale over the image of $\tilde{X}^{\circ}$. Since $\psi$ is finite and the complementary of the image of $\tilde{X}^{\circ}$ in $X$ has codimension at least two, the morphism $\psi$ is a quasi-étale cover of $X$. By some basic properties of the reflexive sheaves and by [10, Proposition 6.9], the following chain of isomorphisms holds

$$
\begin{aligned}
\overline{H^{1}\left(Y, \mathcal{O}_{Y}\right)} & \simeq H^{0}\left(Y, \Omega_{Y}^{[1]}\right) \simeq H^{0}\left(\tilde{B} \times F, \Omega_{\tilde{B} \times F}^{[1]}\right) \\
& \simeq H^{0}\left(\tilde{B}, \Omega_{\tilde{B}}^{[1]}\right) \oplus H^{0}\left(F, \Omega_{F}^{[1]}\right) \neq 0 .
\end{aligned}
$$

So $Y$ is a quasi-étale cover of $X$ that has positive irregularity and this is a contradiction.

\section{Applications and remarks}

In this section we talk about some applications of Theorem 0.4. We start noting the following fact, that will be useful for what follows.

Remark 4.1. In Theorem 0.4, the condition on the augmented irregularity is needed only to avoid the case $X$ is isomorphic in codimension one to an orbibundle. More precisely the hypothesis $\tilde{q}(X)=0$ in Theorem 0.4 (and also in the following corollaries) can be replaced by the following weaker condition: $X$ is not isomorphic in codimension one over $B$ to a quasi-étale quotient of $\tilde{B} \times E$ for some finite cover $\tilde{B} \rightarrow B$.

We should briefly remark that a careful analysis of rational curves on orbibundles, can give a better understanding of the problem. 
Remark 4.2. The condition of not being an orbibundle is sufficient to have a divisor covered by vertical rational curves, but it is not necessary. Indeed it is not difficult to show that an orbibundle $\tilde{B} \times E \stackrel{f}{\rightarrow} X_{\text {orb }}$ contains a divisor covered by vertical rational curves if and only if $f$ is not étale in codimension two. In particular this observation provides a characterization of those relatively minimal Calabi-Yau fiber spaces with a divisor covered by vertical rational curves.

Returning to Question 0.2, we can give a complete answer (for vertical rational curves) in the case of a relatively minimal genus-one fibration.

Theorem 4.3. Let $X \stackrel{\psi}{\rightarrow} B$ be a relatively minimal genus-one fibration. Then $X$ does not contain vertical rational curves if and only if there is a finite globally étale cover of $X$ isomorphic to $\tilde{B} \times E$ over $B$, for some finite cover $\tilde{B}$ of $B$.

Proof. $(\Leftarrow)$ Suppose there is a finite cover $\tilde{B} \rightarrow B$ such that we have the following diagram

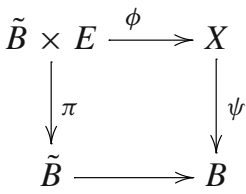

where $\phi$ is finite and globally étale. The restriction of $\phi$ to any fiber $\pi^{-1}(t)$ is étale, so the image in $X$ is again a genus-one curve in $X$. Any fiber of $\psi$ is the image of a curve obtained in this way, so all the vertical curves have genus equals to one.

$\Rightarrow)$ Since $\psi$ is relatively minimal the exceptional locus of $\psi$ is covered by rational curves contracted by $\psi$ by [15]. So we can suppose that $\psi$ is equidimensional. Moreover by Lemma 3.5 we can assume that $\psi$ is isotrivial. Under these conditions we can apply [17, Theorem 44 (1)] and obtain that $X$ is birational to a unique orbibundle $X_{\text {orb }}$.

Let us consider the following diagram

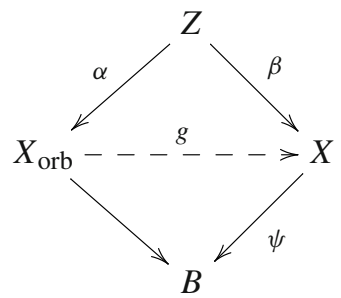

where $g$ is the rational map given by [17, Theorem 44 (1)] and $Z$ is a resolution of the morphism. Since $\psi$ is a relatively minimal Calabi-Yau fiber space by $[1$, Theorem 0.2$]$ there exists some boundary $\Delta$ on $B$ such that $(B, \Delta)$ is klt. By [18, Proposition 5.20] also $\left(X_{\mathrm{orb}}, \Delta^{\prime}\right)$ is klt for some boundary. Finally, applying [13, Corollary 1.7] to $g$, we deduce that the indeterminacy locus of $g$ is covered by rational curves. 
We claim that such curves must be vertical. Indeed if the $\beta$-exceptional locus contains the $\alpha$-exceptional locus, then $g$ is globally defined by [6, Lemma 1.15]. Suppose this inclusion does not hold and denote by $p \in X_{\text {orb }}$ a point in the image of $\operatorname{Exc}(\alpha) \backslash \operatorname{Exc}(\beta)$. By [13, Corollary 1.6] $\alpha^{-1}(p)$ is rationally connected. Choose two points in $\alpha^{-1}(p)$ that maps into different points of $X$ and take a rational curve $R$ passing through these points. Its image $\beta(R)$ is a rational curve in $X$ and, since the diagram above commutes, it must be contracted in $B$, that proves the claim.

Assume that $X$ does not contain vertical rational curves. Since all the fibers of the orbibundle are irreducible curves, by dimensional reasons, $g$ cannot contract anything, and hence $X$ is isomorphic to $X_{\text {orb }}$. If the finite map $\tilde{B} \times E \stackrel{h}{\rightarrow} X$ ramifies at some point $(b, z)$ then the map restricted to the fiber $b \times E \rightarrow X$ is a finite ramified map from a genus one curve onto its image, that by Hurwitz formula is a genus-zero curve that is contracted in $B$. Hence $h$ is globally étale.

Remark 4.4. In the first version of this theorem, there was the assumption of $\mathbb{Q}$ factoriality both on $B$ and $X$. This assumption was used in order to apply [17, Theorem 44 (2)] and obtain directly an isomorphism between $X$ and $X$ orb. According to [17, Theorem 44 (2)], under some assumptions on the regularity of $X$ and $B$, the variety $X$ is isomorphic to an orbibundle. Unfortunately, it turns out that this part of the theorem is false also with the further assumptions that $X$ and $B$ are smooth projective varieties. The heuristic reason for the failure of this result is the possible existence of divisors of insufficient fiber type in the fibration $X \rightarrow B$ that can be contracted in $X_{\text {orb }}$.

The standard construction of a K3 surface of Kummer type gives a counterexample: let $E$ be an elliptic curve. The quotient $(E \times E) / \pm$ under the diagonal action of the involution, which fibres over $\mathbb{P}^{1}$, is an orbibundle. However, its minimal resolution is a smooth $\mathrm{K} 3$ surface with an induced isotrivial fibration, that is equidimensional and relatively minimal but it is not isomorphic to an orbibundle.

Now we present some applications of our results.

Corollary 4.5. In the setting of Theorem 4.3, if B does contain no rational curves, then $X$ contains rational curves if and only if there exists no finite étale cover of the form $\tilde{B} \times E \rightarrow X$ over $B$.

Corollary 4.6. Let $X$ be a projective variety of dimension $n$ with log terminal singularities, $\kappa(X)=n-1$ and $\tilde{q}(X)=0$. The variety $X$ does contain rational curves.

Proof. If the canonical bundle of $X$ is not nef, by the Cone Theorem then there are rational curves in $X$. The numerical dimension of $K_{X}$ is greater than the Kodaira dimension. If $v\left(K_{X}\right)=n$, then the canonical bundle is big and $X$ is of general type, that is a contradiction. So $v\left(K_{X}\right)=n-1=\kappa\left(K_{X}\right)$ and the canonical bundle is semi-ample. In particular the Iitaka fibration of the canonical bundle gives a genusone fibration $\varphi_{K_{X}}: X \rightarrow B$ with $K_{X} \sim_{\mathbb{Q}} \varphi_{K_{X}}^{*}(H)$ for a $\mathbb{Q}$-Cartier $\mathbb{Q}$-divisor $H$ on $B$. In particular we can apply Theorem 0.4 and get the thesis.

We state Theorem 0.4 without boundary. 
Corollary 4.7. Let $X \rightarrow B$ be a relatively minimal Calabi-Yau fiber space with $\operatorname{dim}(X)=\operatorname{dim}(B)+1$ and $\tilde{q}(X)=0$. Then there exists a subvariety of $X$ of codimension one covered by rational curves contracted in $B$.

This result is a generalization of [2, Theorem 1]. Also for smooth varieties this theorem improves the results of [7], because we can certainly apply Corollary 4.7 to smooth varieties with finite fundamental group. Corollary 4.7 can be generalized in the case of non relatively minimal genus-one fibration getting the following statement.

Proposition 4.8. Let $X \stackrel{\phi}{\rightarrow} B$ be a genus-one fibration. Suppose that $X$ has klt singularities, $\tilde{q}(X)=0$ and $K_{X} \sim_{\mathbb{Q}} \phi^{*} L+\sum a_{i} E_{i}$ for some exceptional divisors. If we suppose that some $a_{i}$ is non-negative, then $X$ does contain a uniruled divisor.

Proof. If there are no exceptional divisors we can apply Theorem 0.4 to conclude. Since we are looking for a uniruled divisor and a $\mathbb{Q}$-factorialization is small we can assume that $X$ is $\mathbb{Q}$-factorial. We can write $K_{X} \sim_{\mathbb{Q}} \phi^{*} L+\sum a_{i} E_{i}-\sum b_{i} F_{i}$ with $E_{i} \neq F_{j}$ the exceptional divisors and with all the coefficients non-negative. The divisor $\sum a_{i} E_{i}$ has a component $E_{1}$ covered by curves $C_{t}$ contracted in $B$ such that $\sum a_{i} E_{i} \cdot C_{t}<0$ by [19, Lemma 2.9]. Moreover the curves $C_{t}$ are complete intersections of the form $E_{1} \cap H_{1} \cap \cdots \cap H_{n-2}$ for some base points free divisors $H_{i}$ in $X$.

Take a terminalization $\tilde{X} \stackrel{v}{\rightarrow} X$. The canonical bundle of this partial resolution is $K_{\tilde{X}} \sim \mathbb{Q} v^{*} K_{X}-\sum c_{i} G_{i}$ for some non negative number $c_{i}$. A component of the divisor $v^{*} E_{1}$, that is $\left(v^{-1}\right)_{*} E_{1}$, is covered by the strict transforms $\tilde{C}_{t}$ and satisfies $\sum a_{i} v^{*} E_{i} \cdot \tilde{C}_{t}<0$. The family of curves $\tilde{C}_{t}$ is not contained in the other components of the support of $K_{\tilde{X}}$, so we have $K_{\tilde{X}} \cdot \tilde{C}_{t}<0$. Since $\tilde{X}$ is terminal, it is smooth in codimension 2 . The curves $\tilde{C}_{t}$ are contained in the intersection $v^{*} H_{1} \cap \cdots \cap v^{*} H_{n-2}$. The divisors $v^{*} H_{i}$ are base point free, so the general element of this family does not intersect the singular points of $\tilde{X}$. This means that for a general point in $\left(v^{-1}\right)_{*} E_{1}$ there is a curve contained in the regular part of $X$ that intersects negatively the canonical bundle $K_{\tilde{X}}$, hence we can apply [6, Theorem 3.6] and get a family of rational curves that covers $\left(v^{-1}\right)_{*} E_{1}$. Since the image of a rational curve is again rational, this implies that also $E_{1}$ is covered by rational curves.

For smooth varieties we can do slightly better. The key point for this improvement is a very nice result for varieties covered by elliptic curves by Lazic and Peternell [20, Theorem 6.12]. For smooth varieties we can prove, using their result, the following corollary.

Corollary 4.9. Let $X$ be a smooth projective variety with $\tilde{q}(X)=0$. If $X$ is covered by genus-one curves, then it contains a rational curve.

Proof of Theorem 0.6. Suppose by contradiction that $X$ does not contain rational curves. We can apply [20, Theorem 6.12] and find an equidimensional fiber space $X \rightarrow W$. This fibration is relatively minimal and we can proceed as in the proof of Theorem 0.4 and find an irregular quasi-étale cover of $X$. 
In particular this proves the following result.

Corollary 4.10. Let $X$ be a smooth projective variety covered by elliptic curves but with no rational curves. Then the fundamental group of $X$ is infinite.

We state a useful criterion to find elliptic fibrations due to Kóllar.

Theorem 4.11. Let $X$ be a projective variety of dimension $n$ with klt singularities, nef canonical bundle and $L$ a Cartier divisor on X. Assume moreover

(1) $L^{n-2} \cdot \operatorname{Td}_{2}(X)>0$.

(2) $L$ is nef.

(3) $L-\varepsilon K_{X}$ is neffor $0 \leq \varepsilon<<1$.

(4) $L^{n}=0$.

(5) $L^{n-1} \neq 0$ in $H^{2 n-2}(X, \mathbb{Q})$.

Then $X$ with the Iitaka fibration associated to $L$ is a relatively minimal genus-one fibration.

This result is [17, Theorem 10]. In the same article there is also a log version of this theorem.

Remark 4.12. In [17, Theorem 10] there is the further hypothesis $L^{n-1} \cdot K_{X}=0$, but this condition actually follows from the others. Indeed if $L-\varepsilon K_{X}$ is nef then

$$
0 \leq\left(L-\varepsilon K_{X}\right)^{n}=L^{n}-n \varepsilon L^{n-1} \cdot K_{X}+\cdots=-n \varepsilon L^{n-1} \cdot K_{X}+\cdots
$$

The divisors $L$ and $K_{X}$ are nef, hence $L^{n-1} \cdot K_{X} \geq 0$. It follows that $L^{n-1} \cdot K_{X}=0$. It follows from Theorem 4.11 the following result.

Corollary 4.13. Let $X$ be a variety with log terminal singularities and $\tilde{q}(X)=0$. If there exists a line bundle $L$ on $X$ such that the conditions from 1 to 5 of Theorem 4.11 are satisfied, then $X$ does contain rational curves.

Let us conclude with an example of a threefold which is regular and admits a genus-one fibration, but it does not contain rational curves.

Example 4.14. Consider in $\mathbb{P}^{3}$ a general hypersurface $S$ of degree at least 5. By [5] there are no rational curves in $S$. There is a $2: 1$ cover $\tilde{S}$ of $S$ ramified along the intersection between $S$ and a quadric that is constructed as a complete intersection in $\mathbb{P}^{4}$. This cover comes with an involution $\eta \in \tilde{S}$ such that $S$ is the quotient of $\tilde{S}$ by this involution. Consider the order two automorphism $\alpha$ of the product $Y:=\tilde{S} \times(\mathbb{C} /(\mathbb{Z} \cdot 1 \oplus \mathbb{Z} \cdot i))$ defined by $\alpha(x, z)=\left(\eta(x), \frac{1+i}{2}-z\right)$. The quotient of $Y$ under this action gives a genus-one fibration $X:=Y / \alpha \stackrel{\pi}{\rightarrow} S$. The quotient map $Y \rightarrow X$ is globally étale, that implies that all the fibers of $\pi$ are genus-one curves. The holomorphic 1-forms on $X$ are exactly the holomorphic 1-forms on $Y$ that are invariant under the action of $\alpha$. Since $\tilde{S}$ is a complete intersection in $\mathbb{P}^{4}$, it is regular. A 1-forms $\omega$ on $Y$ can be written as $\pi^{*} \beta$ where $\pi$ is the projection from $Y$ to $E:=\mathbb{C} /(\mathbb{Z} \cdot 1 \oplus \mathbb{Z} \cdot i)$. By the choice of the automorphism on $E$ we see that $\alpha^{*} \omega=-\omega$, hence $q(X)=0$. Since all the vertical curves have genus one and an horizontal rational curve gives a rational curve on $S$, there are no rational curves on $X$. So this is an example of a genus-one fibration from a variety with no rational curves and irregularity equals to 0 . 
Acknowledgements The author warmly thanks Raffaele Carbone for the continuous helpful discussion during the writing of this paper and Andreas Höring for many useful comments. The author would also like to thank his advisor, Simone Diverio, for the continuous help provided during this work.

Funding Open access funding provided by Università degli Studi Roma Tre within the CRUI-CARE Agreement.

Open Access This article is licensed under a Creative Commons Attribution 4.0 International License, which permits use, sharing, adaptation, distribution and reproduction in any medium or format, as long as you give appropriate credit to the original author(s) and the source, provide a link to the Creative Commons licence, and indicate if changes were made. The images or other third party material in this article are included in the article's Creative Commons licence, unless indicated otherwise in a credit line to the material. If material is not included in the article's Creative Commons licence and your intended use is not permitted by statutory regulation or exceeds the permitted use, you will need to obtain permission directly from the copyright holder. To view a copy of this licence, visit http://creativecommons.org/ licenses/by/4.0/.

\section{References}

[1] Ambro, F.: The moduli b-divisor of an lc-trivial fibration. Compos. Math. 141(2), 385403 (2005)

[2] Anella, F.: Rational curves on fibered varieties. Mathematische Zeitschrift, 1-15 (2020)

[3] Barth, W.P., Hulek, K., Peters, C.A.M., Van de Ven, A.: Compact Complex Surfaces, Volume 4 of Ergebnisse der Mathematik und ihrer Grenzgebiete, 2nd edn. SpringerVerlag, Berlin (2004)

[4] Catanese, F.: Q.E.D. for algebraic varieties. J. Differ. Geom. 77(1), 43-75 (2007)

[5] Clemens, H.: Curves on generic hypersurfaces. Ann. Scientifiques de l'École Normale Supérieur 19, 629-636 (1986)

[6] Debarre, O.: Higher-dimensional Algebraic Geometry, Universitext. Springer-Verlag, New-York (2001)

[7] Diverio, S., Fontanari, C., Martinelli, D.: Rational curves on fibered Calabi-Yau manifolds. Doc. Math. 24, 663-675 (2019)

[8] Druel, S.: A decomposition theorem for singular spaces with trivial canonical class of dimension at most five. Invent. Math. 211(1), 245-296 (2018)

[9] Greb, D., Guenancia, H., Kebekus, S.: Klt varieties with trivial canonical class: holonomy, differential forms, and fundamental groups. Geom. Topol. 23(4), 2051-2124 (2019)

[10] Greb, D., Kebekus, S., Peternell, T.: Singular spaces with trivial canonical class. In: Kollár, J., Fujino, O., Mukai, S., Nakayama, N. (eds.) Minimal Models and Extremal Rays (Kyoto, 2011). Volume 70 of Adv. Stud. Pure Math., pp. 67-113. Math. Soc. Japan, Tokyo (2016)

[11] Grothendieck, A.: Élements de géométrie algébrique III: Étude cohomologique des faisceaux cohérents, première partie. Publications Mathématiques de l'IHÉS 11, 5167 (1961)

[12] Hartshorne, R.: Deformation Theory, vol. 257. Springer, Berlin (2010)

[13] Hacon, D.C., McKernan, J.: On Shokurov's rational connectedness conjecture. Duke Math. J. 138(1), 119-136 (2007) 
[14] Höring, A., Peternell, T.: Algebraic integrability of foliations with numerically trivial canonical bundle. Invent. Math. 216(2), 395-419 (2019)

[15] Kawamata, Y.: On the length of an extremal rational curve. Invent. Math. 105(1), 609$611(1991)$

[16] Kodaira, K.: On compact analytic surfaces: II. Ann. Math. 77, 563-626 (1963)

[17] Kollár, J.: Deformations of elliptic Calabi-Yau manifolds. Recent Adv. Algebr. Geom. 417, 254-290 (2015)

[18] Kollár, J., Mori, S.: Birational Geometry of Algebraic Varieties. Cambridge University Press, Cambridge (1998)

[19] Lai, C.: Varieties fibered by good minimal models. Math. Ann. 350(3), 533-547 (2011)

[20] Lazić, P., Peternell, T.: Maps from K-trivial varieties and connectedness problems. Annales Henri Lebesgue 3, 473-500 (2020)

[21] Voisin, C.: On some problems of Kobayashi and Lang: algebraic approaches. Curr. Dev. Math. 2003, 53-125 (2003)

Publisher's Note Springer Nature remains neutral with regard to jurisdictional claims in published maps and institutional affiliations. 